\title{
Extended Talairach Landmarks on Neuroimages for Atlas Registration
}

\author{
Guoyu Qian ${ }^{1}$, Suhuai Luo ${ }^{1}$, Jesse Jin ${ }^{1}$, Wieslaw L. Nowinski ${ }^{2}$ \\ ${ }^{1}$ School of Design, Communication and IT, The University of Newcastle, Australia \\ ${ }^{2}$ Biomedical Imaging Lab, Agency for Science, Technology and Research, Singapore
}

\begin{abstract}
The accuracy of scan-to-atlas registration highly depends on the number of landmarks and the precision of landmark identification. An extended landmark, cerebellum inferior (CBI), is introduced in this paper. The extracted brain and midsagittal plane are applied to identify the modified Talairach landmarks and the new introduced landmark CBI. The AC-PC plane is firstly determined and then anatomical information is applied to estimate the other landmarks. The proposed method is fully automatic and has been validated on 49 FDG-PET normal and pathological scans qualitatively, and 15 cases quantitatively. The average processing time is about 3 seconds on a standard personal computer.
\end{abstract}

Keywords-Talairach; landmarks; atlas; transformation

\section{INTRODUCTION}

Atlas-assisted operations on medical images of human brain are widely applied to image segmentation [1, 2], data normalization [3], and localization analysis [4-6], especially for the positron emission tomography (PET) images with a poorer spatial resolution and lower signal-noise ratio than other modalities like magnetic resonance imaging or computed tomography. PET is a nuclear medicine imaging technique to produce a three dimension (3D) image of functional processes in the body, by a tracer injected into a patient. Fluoro-deoxyglucose (FDG) is a commonly used tracer to distinguish the high-glucose-consuming cells, e.g. the cerebrum and cerebellum, from the other parts of the body [7]. This imaging technique is increasingly used for detection and diagnosis of tumor and neurological diseases, e.g. Alzheimer's disease.

There are numerous printed and electronic brain atlases have been developed $[8,9]$. Talairach and Tournoux introduced a brain atlas [10], which is commonly used as a reference brain and a gold standard in human brain mapping. The original Talairach landmarks contain two subcortical landmarks, anterior commissure $(A C)$ and posterior commissure $(P C)$, and six cortical landmarks. However, the original Talairach landmarks have several limitations and problems, e.g. not all of the landmarks are available on the Talairach atlas. A set of modified Talairach landmarks, conceptually equivalent to the original Talairach landmarks, was introduced [8] to overcome the problems and become more constructive and easier to be identified by computer program. The modified Talairach landmarks are automatically identified on magnetic resonance neuroimages $[11,12]$, and applied to the Talairach transformation [4] and other atlas-assisted automatic interpretations and applications $[5,6]$.

In this paper, a new landmark is introduced for the cerebellum inferior $(C B I)$, which is at the most inferior point of the cerebellum, to enclose the cerebellum into the Talairach space for rapid Talairach transformation. After adding the new landmark, the whole brain will be subdivided into 18 cuboidal regions including both cerebrum and cerebellum (as opposed to the original 12 cuboids) by 9 landmarks - CBI plus 8 modified Talairach landmarks.

\section{BACKGROUND}

\section{A. Original and Modified Talairach Landmarks}
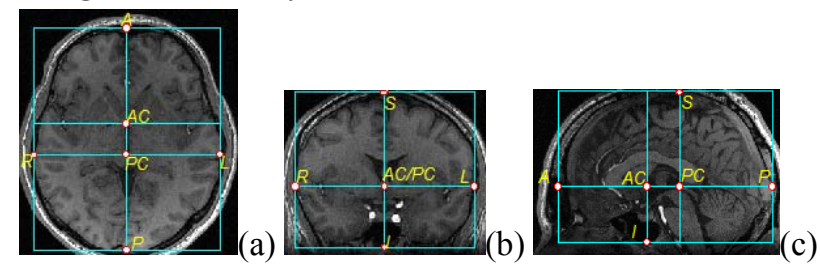

Figure 1. Modified Talairach landmarks on a) axial; b) coronal; and c) sagittal orientations.

\section{1) Original Talairach Landmarks}

The original Talairach landmarks define two internal landmarks $A C$ and $P C$, and six external landmarks $L, R, A, P$, $S$, and $I[10] . A C$ is the point of intersection of the lines passing through the superior edge of the anterior commissure and the posterior edge of the anterior commissure. $P C$ is the point of intersection of the lines passing through the inferior edge of the posterior commissure and the anterior edge of the posterior commissure. The external landmarks are the points on the cortex. They are: the most superior point of the parietal cortex $(S)$ and the most inferior point of the temporal cortex $(I)$; the most anterior point of the frontal cortex $(A)$ and the most posterior point the occipital cortex $(P)$; the most lateral points (left and right) of the parietotemporal cortex ( $L$ and $R$ ). The original Talairach landmarks have some limitations and several problems such as, not all of the landmarks are available in the original brain atlas; the cortical landmarks are not defined in a constructive way; the intercommissural landmarks are located beyond their own structures; etc.

\section{2) Modified Talairach Landmarks}

The modified Talairach landmarks [8] define $A C$ and $P C$ within the midsagittal plane (MSP), and are the central points 
of the anterior commissure and posterior commissure respectively. The landmarks $A, P, L$ and $R$ are identified from AC-PC plane passing through both $A C$ and $P C$; the landmark $I$ is identified on a coronal plane passing through $A C$ (VAC); and the landmark $S$ is identified on another coronal plane passing through $P C$ (VPC). Fig. 1 shows the modified landmarks on axial, coronal and sagittal orientations. The whole brain is subdivided into 12 cuboids.

\section{MATERIALS AND METHODS}

\section{A. Extended Talairach Landmark}

The extended Talairach landmark is defined to include the cerebellum of a brain into the Talairach space. Similarly to the cerebrum, the cerebellum has the cerebellar cortex which is a thin outer layer of gray matter. The most inferior point of the cerebellum is defined as a new landmark $C B I$, as shown in Fig. 2. For more constructive way of automatic identification, the landmark $C B I$ is defined as the point of intersection of three planes: MSP, the coronal plane passing through the most superior (most dorsal) point of the cerebellum, and the axial plane passing through the most inferior (most ventral) point of the cerebellar cortex. By adding the $C B I$ landmark, the Talairach grid system is increased to 18 cuboids. Both the cerebrum and cerebellum are included into the Talairach space as shown in Fig. 3.

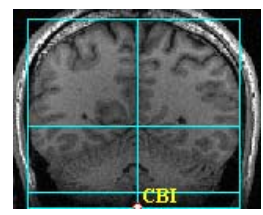

Figure 2. Extended Talairach landmark on a coronal slice.

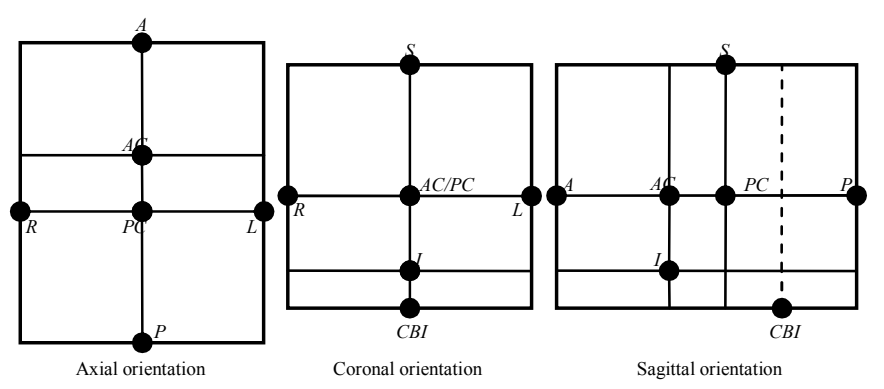

Figure 3. Grid system with the extended Talairach landmark.

\section{B. Materials}

The proposed approach of automatic landmark placement was evaluated with the FDG-PET images from the Alzheimer's Disease Neuroimaing Initiative (ADNI) database. Each case has 6 dynamic frames with scanning every 5 minutes from 30 to 60 minutes after FDG injection. Those scans were preprocessed by the following steps: 1) registered to the first frame, 2) averaged, 3) reoriented into a grid of $160 \times 160 \times 96$ voxels with voxel size $1.5 \times 1.5 \times 1.5 \mathrm{~mm}$ [13]. The preprocessed images have their horizontal axis paralleled with the anterior commissure and posterior commissure.

\section{Methods}

The automatic approach of landmark placement is based on our previous work on brain extraction and midsagittal lines detection from FDG-PET images [14] shown in Fig. 4. The proposed method comprises two parts: 1) the modified Talairach landmarks identification; and 2) the extended landmark $C B I$ placement on FDG-PET images.
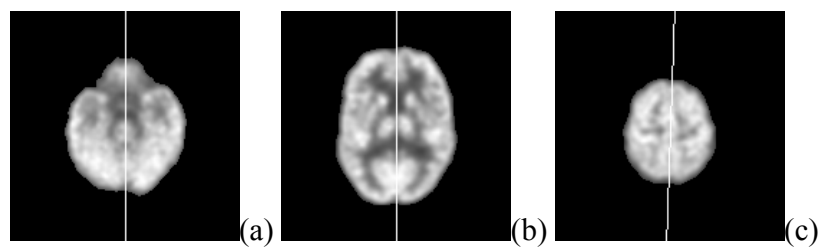

Figure 4. Extracted brain from FDG-PET images with MSP drawing. (a) inferior slice; (b) middle slice; and (c) superior slice.

\section{Detection of the modified Talairach landmarks}

The modified Talairach landmarks are detected by the following steps: a) detect AC-PC line (APL) on MSP; b) determine $L, R, A, P$ landmarks on AP plane; c) determine $I$ on VAC plane, and decide $A C$ position; and d) determine $S$ on VPC plane, and decide $P C$ position.

\section{a) Detect $A C-P C$ line ( $A P L)$ on MSP}

APL is an intersection line of MSP and AC-PC plane, which is a perpendicular plane to MSP and passes through both the $A C$ and $P C$. The detection of APL is the first and important step to extract the AC-PC plane and affect the results of the subsequent steps to detect the other landmarks. Since FDGPET images lack structural information, it is difficult to detect the anterior commissure and posterior commissure structure on the images directly as well as the $A C$ and $P C$ landmarks. An alternative way to estimate the APL from MSP is to detect the longest line from anterior to posterior (Fig. 5) on the MSP image with skull removed. Even though the exact positions of $A C$ and $P C$ are still unsure, they will be determined later by the inferior and superior landmarks according to the modified Talairach space.
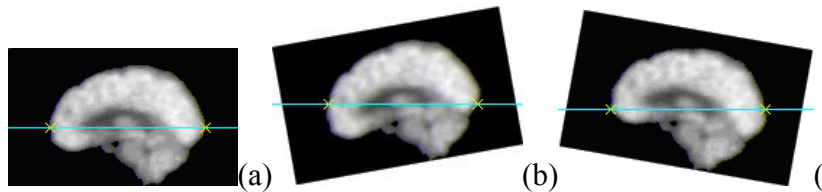

(b)

Figure 5. AC-PC line on MSP. (a) $\lambda=0^{\circ}$; (b) $\lambda=-10^{\circ}$; (c) $\lambda=+10^{\circ}$.

The algorithm to detect the longest line from MSP is by searching the projections from both anterior and posterior directions. On a 2D image of MSP (Fig. 5), the searching is from both the left and the right of the image. Since APL has its anatomical position within human brain, the proportional scaling in the Talairach space (e.g. the distance between $A$ and $P$ is $174 \mathrm{~mm}$, the distance between $S$ and APL is $74 \mathrm{~mm}$, and the distance between $I$ and APL is $43 \mathrm{~mm}$ ) is applied to the search algorithm. After the detection of the longest horizontal line, the MSP image needs to be rotated through a degree of $\lambda$ (e.g. $10^{\circ}$ ) to find out the longest line from non-horizontal direction. It can be done by a loop of rotation from $-\lambda$ to $+\lambda$ with step $1^{\circ}$. 


\section{b) Determine $L, R, A, P$ on $A C-P C$ plane}

AC-PC plane (Fig. 6) is extracted from the volumetric data based on APL and the skull-stripped brain [14]. The algorithm to determine the $L$ and $R$ landmarks is to select the extreme left point and extreme right point as $L$ and $R$ landmarks by searching the lines parallel to MSP. Similarly, to determine the $A$ and $P$ landmarks, the extreme anterior point and extreme posterior point are selected by searching the lines perpendicular to MSP [14]. Fig. 6 shows the AC-PC plane with $L, R, A, P$ landmarks identified.

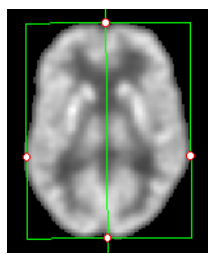

Figure 6. AC-PC plane with $L, R, A, P$ landmarks

\section{c) Determine I and AC}

In the Talairach space, the length from anterior to posterior is $174 \mathrm{~mm}$. The distances from anterior to $A C$ and $P C$ are $70 \mathrm{~mm}$ and $94 \mathrm{~mm}$, respectively. They are about $40 \%$ and $54 \%$ of the whole line. According to the definition of the Talairach space and the positions of $A$ and $P$, the $A C$ and $P C$ positions are estimated by proportional scaling. They are marked as estimated-AC (eAC) and estimated-PC (ePC). The coronal slices near eAC (e.g. $\pm 5 \mathrm{~mm}$ ) are selected as the candidates of VAC. By calculating the most inferior point on all VAC candidates, the $\mathrm{VAC}$ is identified and the $A C$ is also determined at the same time. Fig. 7a shows an example of $I$ on VAC.

\section{d) Determine $S$ and $P C$}

Similarly, the coronal slices near ePC (e.g. $\pm 5 \mathrm{~mm})$ are selected as the candidates of VPC. By calculating the most superior point on all VPC candidates, the VPC is identified and the $P C$ is also determined at the same time. Fig. $7 \mathrm{~b}$ shows an example of $S$ on VPC, and Fig. 7c shows $A C$ and $P C$ on MSP.
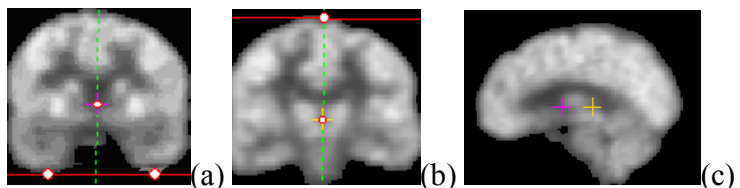

Figure 7. $I, S, A C$ and $P C$ landmarks. (a) $I$ landmark on VAC; and (b) $S$ landmark on VPC; (c) $A C$ and $P C$ on MSP.

Determine the extended landmark CBI on FDG-PET images

A coronal slice passing through the most superior point of cerebellum is called VCB. The extended landmark $C B I$ will be located by the following steps: a) estimate the position of VCB $(\mathrm{eVCB})$ and select the candidates of VCB; and b) Locate $C B I$ from the candidates of VCB.

\section{a) Estimate the position of $V C B(\mathrm{eVCB})$ and select the candidates of $V C B$}

In order to estimate the position of $\mathrm{VCB}$, a transverse fissure cerebellar tentorium, is detected first. It is located between cerebrum and cerebellum [15]. According to the FDG-
PET image property of that the cerebrum and cerebellum have much greater glucose uptake values than the other fissures [16, 17], the fissure between cerebrum and cerebellum is detected by the following steps:

Define a region-of-interest (ROI) for faster detection of cerebellar tentorium. The ROI of $\mathrm{X}$-axis starts from $P C$ and ends at $P$. The ROI of $\mathrm{Y}$-axis starts from APL and ends at the bottom of MSP image. All the processing and calculation below are restricted within this ROI (Fig. 8a).

Define a glucose consumption line (GCL), starting from the top edge of ROI, ending at the cortical surface of brain.

$$
y=\operatorname{tg} \theta \times\left(x-x_{0}\right)
$$

where $\theta$ is the angle between GCL and X-axis, and $\mathrm{x}_{0}$ is the position on AC-PC line.

Calculate the sum of glucose consumption on GCL.

$$
S\left(x_{0}, \theta\right)=\sum G C L\left(x, y, x_{0}, \theta\right)
$$

where $\mathrm{x}$ and $\mathrm{y}$ are the coordinates of GCL on MSP image.

Locate a GCL with minimum sum of glucose consumption $\mathrm{GCL}_{\min }$.

The eVCB is identified as the cross point of $\mathrm{GCL}_{\min }$ and APL (Fig. 8b). The coronal slices passing through and nearby eVCB (e.g. $\pm 3 \mathrm{~mm}$ ) are selected as the candidates of VCB.
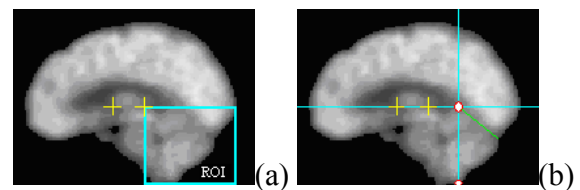

Figure 8. Estimated VCB plane. (a) ROI definition; (b) GCL calculation

\section{b) Locate CBI from the candidates of $V C B$}

The cerebellum has two hemispheres connected by the vermis. There are two conditions need to be considered while calculating the lowest part on coronal images. The most inferior bottom points of cerebellum are lower than the vermis and higher than the brainstem. As the size of the brainstem is wider than that of the vermis, the width about $7 \mathrm{~mm}$ is excluded from both hemisphere sides for calculation of cerebellum bottom. The $C B I$ is located by the following steps:

- Exclude the areas which may contain the brainstem and vermis from the coronal images.

- Detect the lowest part on both hemispheres and calculate the average.

- Find out a slice with lowest average value of the cerebellar hemispheres. The slice is VCB, and the most inferior point on VCB is the landmark $C B I$.

\section{RESULTS}

The proposed approach of automatic landmark placement on FDG-PET images was implemented in $\mathrm{C}++$ running on a standard personal computer with $2.4 \mathrm{GHz} \mathrm{CPU}$. The average processing time was 2.78 seconds per case. A total of 49 cases 
with a resolution of $160 \times 160 \times 96$ voxels of size $1.5 \mathrm{~mm}^{3}$ were tested. Fifteen of them have the landmarks manually placed by a neuroanatomy expert for comparison with the results of the automatic method. Fig. 9 gives an example of grid by detected landmarks on three orientations of FDG-PET images. The other cases were visually checked with satisfactory results.
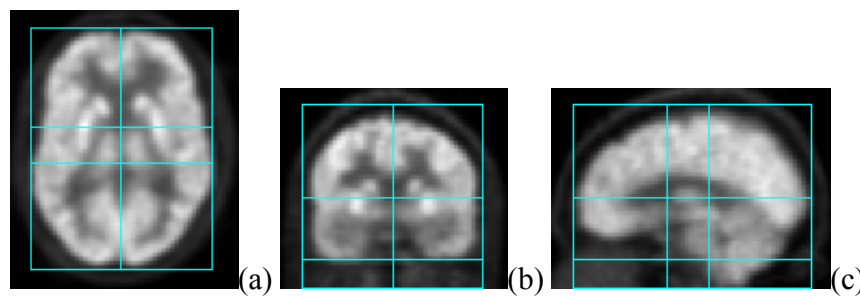

Figure 9. An example of FDG-PET images with grid by detected landmarks. (a) grid on axial slice; (b) grid on coronal slice; and (c) grid on sagittal slice.

Table 1 lists the differences of 15 cases, and their minimum and maximum values, mean and the standard deviations (SD) of the displacements of comparing the automated landmark identification and the manual method for the modified Talairach landmarks $A C, P C, L, R, A, P, S, I$, and $C B I$. The differences of other landmarks are the simple absolute difference value of ground truth and automatic approach. From the result table, there is no significant statistical difference was observed.

TABLE I. TEST RESULTS OF LANDMARK MISPLACEMENT (MILLIMETER)

\begin{tabular}{|c|c|c|c|c|}
\hline & $\min$ & $\max$ & $\mu$ & $\sigma$ \\
\hline$A C$ & 0.32 & 2.56 & 1.29 & 0.32 \\
\hline$P C$ & 0.47 & 3.31 & 1.65 & 0.71 \\
\hline$L$ & 0 & 2.27 & 0.72 & 0.47 \\
\hline$R$ & 0 & 2.07 & 0.44 & 0.36 \\
\hline$A$ & 0 & 1.73 & 0.58 & 0.33 \\
\hline$P$ & 0 & 1.38 & 0.38 & 0.2 \\
\hline$S$ & 0 & 2.09 & 0.65 & 0.44 \\
\hline$I$ & 0.38 & 5.72 & 1.81 & 1.66 \\
\hline$C B I$ & 0 & 3.36 & 1.17 & 0.9 \\
\hline
\end{tabular}

\section{CONCLUSION}

In conclusion, a new landmark is introduced to extend the modified Talairach landmarks in order to include cerebellum into the Talairach space and grid system. A fast and fully automatic approach to identify those landmarks from FDGPET images is presented. It has been applied to 49 cases and shown promising results. Although the proposed approach has several limitations, we believe that our method is useful by providing an automatic Talairach transformation for FDG-PET images.

\section{ACKNOWLEDGMENT}

The data for this study have been obtained from the Alzheimer's Disease Neuroimaging Initiative (ADNI) database (www.loni.ucla.edu\ADNI).

\section{REFERENCES}

[1] I. N. C. Lawes, T. R. Barrick, V. Murugam, N. Spierings, D. R. Evans, M. Song, and C. A. Clark, "Atlas-based segmentation of white matter tracts of the human brain using diffusion tensor tractography and comparison with classical dissection," NeuroImage, vol. 39, pp. 62-79, 2008 .

[2] O. T. Carmichael, H. A. Aizenstein, S. W. Davis, J. T. Becker, P. M Thompson, C. C. Meltzer, and Y. Liu, "Atlas-based hippocampus segmentation in Alzheimer's disease and mild cognitive impairment," NeuroImage, vol. 27, p. 979?90, 2005.

[3] R. L. Buckner, D. Head, J. Parker, A. F. Fotenos, D. Marcus, J. C. Morris, and A. Z. Snyder, "A unified approach for morphometric and functional data analysis in young, old, and demented adults using automated atlas-based head size normalization: reliability and validation against manual measurement of total intracranial volume," Neuroimage, vol. 23, pp. 724-738, 2004.

[4] W. L. Nowinski, G. Qian, K. N. Bhanu Prakash, Q. Hu, and A. Aziz, "Fast Talairach Transformation for magnetic resonance neuroimages," Journal of computer assisted tomography, vol. 30, p. 629, 2006.

[5] W. L. Nowinski and D. Belov, "Toward atlas-assisted automatic interpretation of MRI morphological brain scans in the presence of tumor," Academic radiology, vol. 12, pp. 1049-1057, 2005.

[6] W. L. Nowinski, G. Qian, K. N. B. Prakash, I. Volkau, W. K. Leong, S. Huang, A. Ananthasubramaniam, J. Liu, T. T. Ng, and V. Gupta, "Stroke Suite: CAD systems for acute ischemic stroke, hemorrhagic stroke, and stroke in ER," Lecture Notes In Computer Science, vol. 4987, pp. 377-386, 2008.

[7] M. Senda, Y. Kimura, and P. Herscovitch, Brain imaging using PET: Academic Press, 2002.

[8] W. L. Nowinski, "Modified Talairach landmarks," Acta Neurochirurgica, vol. 143, pp. 1045-1057, 2001.

[9] A. W. Toga, P. M. Thompson, S. Mori, K. Amunts, and K. Zilles, "Towards multimodal atlases of the human brain," Nature Reviews Neuroscience, vol. 7, pp. 952-966, 2006.

[10] J. Talairach and P. Tournoux, Co-planar stereotaxic atlas of the human brain: Thieme New York, 1988.

[11] K. N. Bhanu Prakash, Q. Hu, A. Aziz, and W. L. Nowinski, "Rapid and automatic localization of the anterior and posterior commissure point landmarks in MR volumetric neuroimages," Academic Radiology, vol. 13, pp. 36-54, 2006.

[12] Q. Hu, G. Qian, and W. L. Nowinski, "Fast, accurate, and automatic extraction of the modified Talairach cortical landmarks from magnetic resonance images," Magnetic Resonance in Medicine, vol. 53, pp. 970976, 2005.

[13] J. B. S. Langbaum, K. Chen, W. Lee, C. Reschke, D. Bandy, A. S. Fleisher, G. E. Alexander, N. L. Foster, M. W. Weiner, and R. A. Koeppe, "Categorical and correlational analyses of baseline fluorodeoxyglucose positron emission tomography images from the Alzheimer's Disease Neuroimaging Initiative (ADNI)," Neuroimage, vol. 45, pp. 1107-1116, 2009.

[14] G. Qian, S. Luo, J. Jin, M. Park, and W. L. Nowinski, "A fast and automatic approach to extract the brain and midsagittal lines from FDGPET head scans," in The 1st International Conference on Information Science and Engineering, Nanjing, China, Dec 18-20, 2009.

[15] K. M. Van De Graaff, Human anatomy, Sixth edition: McGraw-Hill, 2001 .

[16] S. Zincirkeser, E. Sahin, M. Halac, and S. Sager, "Standardized uptake values of normal organs on $18 \mathrm{~F}$-fluorodeoxyglucose positron emission tomography and computed tomography imaging," Journal of international medical research, vol. 35, pp. 231-236, 2007.

[17] Y. Wang, E. Chiu, J. Rosenberg, and S. S. Gambhir, "Standardized uptake value atlas: characterization of physiological 2-deoxy-2-[18 F] fluoro-d-glucose uptake in normal tissues," Molecular Imaging and Biology, vol. 9, pp. 83-90, 2007.

[18] W. Nowinski and K. Prakash, "Dorsoventral extension of the Talairach transformation and its automatic calculation for magnetic resonance neuroimages," Journal of computer assisted tomography, vol. 29, pp. 863-879, 2005. 